\title{
Creativity and Intelligence in Small and Medium Sized Enterprises: The Role of Information Systems
}

\author{
Gurpreet Dhillon ${ }^{1}$, Bernd Carsten Stahl ${ }^{2}$, and Richard Baskerville ${ }^{3}$ \\ ${ }^{1}$ Virginia Commonwealth University, USA \\ ${ }^{2}$ De Montfort University, UK \\ ${ }^{3}$ Georgia State University, USA
}

\section{Introduction}

The work in this volume examines the real-world confluence of several concepts that are too often studied in isolation from each other. Research into creativity too rarely considers the presumed ability of smaller organisations to contribute a large proportion of the innovations introduced into the practical information systems field. There is also far too little research that addresses the notion that smaller organisations more intelligently manage their creativity and innovation, not only in the organisational signal products, but also in structures and processes found in smaller organisations.

Because organisational design itself benefits from creativity and innovation (Cooper, 2000), smaller organisations have an inbuilt advantage purely in terms of the smaller scope required to reorganise in innovative ways to match their projects and products. This smallness in scope also applies to the information systems of smaller organisations. Quite simply, innovations in smaller organisations do not have to diffuse their innovations as far as in larger organisations. The diffusion can happen faster, and without the need for complex knowledge management structures and agencies needed to make innovation happen in larger organisations (Datta, 2007). This difference in scope means that many of the innovation diffusion principles so important to larger organisations and markets, such as those of Rogers (Rogers, 2003), are less critical for the internal creativity of smaller organisations.

There are many good reasons for paying attention to small and medium sized enterprises (SMEs). They constitute the majority of organisations, they are a main source of employment, they are flexible, and they are often creative and innovative. Despite their economic and theoretical relevance, business-oriented research tends to concentrate on large and multinational organisations. This is true in business studies in general as well as in the field of information systems. That state of affairs is unfortunate because SMEs hold the potential to develop novel uses of technology and integrate them in interesting ways in their business processes. At the same time, information systems have the potential to make uniquely important contributions to SMEs, for example by extending markets or providing resources that individual SMEs typically do not have. These IFIP WG 8.2 proceedings aim to address the lacuna in the literature and provide a current discussion of the relationship between SMEs, creativity and innovation, and information systems.

The 8.2 working group is known for its innovative use of theory and its successful attempts to overcome the weaknesses of established approaches. The 2009 conference 
in Portugal aimed at applying those non-conventional ideas to the area of SMEs. The organisers and chairs were curious to find out how the IFIP WG 8.2 community would take to a set of concrete business challenges that emerge from the context of IS use in SMEs. These proceedings show that the community has been successful in incorporating extant literature and developing it in new and interesting ways. In order to discuss the contributions of the authors in a coherent way, we have identified key challenges related to SME research. The papers pertaining to these have been grouped into the sections outlined below. In each section some novel ideas emerged, as we will show in this introduction.

- Creativity and innovation challenges. While many SMEs are creative and innovate partly because of their need to remain competitive, effective use of information and communication technologies helps in defining newer opportunities and sustainability in a marketplace.

- Challenges in ensuring effective governance. While it is easy for many SMEs to overlook the need for effective governance, the importance and need for good internal controls cannot be overstated. Often in the race to achieve high growth, internal control mechanisms get overlooked.

- Challenges in managing knowledge. Because of the very nature of SMEs, knowledge management is a challenge. The owner-manager structure of most SMEs introduces a lot of variance in managing knowledge and in satisfactory sharing of experiences.

- Organizational challenges, power and politics. Cultural differences amongst SMEs and power and politics of managing small firms pose numerous challenges. Foremost of these is the inter-organizational relationship and the role information systems play in facilitating or hindering progress.

\section{Creativity and Innovation Challenges}

It is a cliché that big organizations are bureaucratic and cumbersome, whereas smaller ones are more agile and flexible. There is nevertheless some truth in the observation that small companies can quickly react to changes in their environment and they can easily be redirected to take advantage of changing markets. This flexibility and maneuverability allows them to profit from creativity and be innovative. These views are probably not contentious. They nevertheless leave open a range of questions. What do we mean by these concepts? The definition of an SME is unproblematic because there are political views on this that are relatively straightforward, even if they may leave shades of grey. The situation is significantly different for the other terms involved. What happens if we undertake an analysis of terms such as creativity or innovation? Can one really allude to creativity with regard to an organization? And what does this have to do with technology?

Boden (2000) suggests that the core of creativity is always an idea. If creativity is linked to ideas then one can reasonably argue that the bearers and originators of ideas, namely human beings, can also be creative. It is much less simple to argue, however, that organizations can be the originators of ideas. Creativity of organizations is therefore presumably something different than creativity of individuals. It may be a property 
of an organization or an emerging characteristic of the way in which a social system develops. But where does this leave technology and specifically information systems? Lessig (2001) has argued that the development of the Internet was characterised by creativity and innovation. This leaves open the question whether it was the technology per se that had aspects of creativity or whether it was creativity of the individuals involved in building the technology that led to its phenomenal success.

Despite these and other conceptual uncertainties, innovation is often described as a consequence of creativity and is seen as an essential component of organizations in fast-moving markets. Creativity is seen as a key to organizational survival. While creativity and innovation are certainly important elements, especially for SMEs, to a large extent success at being creative and innovative depends on the market conditions. The chapters in the first section are those that deal directly with the conference theme of creativity and innovation in SMEs. They use a variety of approaches and theoretical lenses to increase our understanding of relevant phenomena.

A fitting starting chapter for this book is the one on "SMEs, IT, and the Third Space: Colonization and Creativity in the Theatre Industry" by Kendall and Kendall. This chapter draws on the rich tradition of social theory employed in the IFIP WG8.2 and uses this tradition to shine an interesting light on a very specific instance of small and medium sized enterprises, namely the management of theatres. Drawing on the concept of the third space, they develop a narrative of knowledge transfer, in particular the transfer of culture. This novel view of knowledge transfer is contextualised with the more established literature on knowledge management. As a result, the authors draw conclusions that allow a better understanding of the mechanisms that allow exchange between large commercial and small not-for-profit theatres.

Valcárcel offers a case study from Germany in her "SME 2.0: Roadmap towards Web 2.0-based Open Innovation in SME-Networks - A Case Study Based Research Framework" This case study is premised on the economic importance of SMEs and their well-known limitations with regards to innovations. The idea she develops and that reoccurs in a number of chapters in this book is that SMEs need to find ways of collaborating in order to ensure that they can make use of innovation. The specific interest of this chapter is the role that Web 2.0 technologies can play in facilitating such collaboration. The communicative features of Web 2.0 technologies are seen as a way of breaking down traditional barriers to knowledge creation and leading to open innovation. This communication can also create marketplaces, foster trust and lead to further collaboration. Valcárcel uses the findings of her case studies to develop a framework for better understanding the processes involved which also provide a basis for future research.

The following chapter on "Conducting Creativity Brainstorming Sessions in Small and Medium-Sized Enterprises Using Computer-Mediated Communication Tools" by Murthy offers a complementary view. Instead of Web 2.0 technologies, it uses computer-mediated communication tools that are in use by SMEs. These tools incorporate systems that are often related to Web 2.0 but in many instances go beyond what private individuals might use. In addition to chat and synchronous meeting facilities, these include electronic brainstorming technologies. Murthy investigates how such tools can be used for optimal results by SMEs. It derives recommendations from a set of experimental studies that require participants to use domain-specific knowledge. The resulting recommendations can be used as practical suggestions for SMEs to optimise their approach towards using brainstorming technologies. 
The subsequent chapter by Nagle and Golden on "An Examination of the Disruptive Innovation Paradox: The Application of the Innovators Dilemma to SMEs" continues the theme of critical questioning of conventional wisdom concerning innovation in SMEs. The authors draw on the literature developed on paradoxes in modern organizations, in particular the innovator's dilemma. They apply a discussion developed in the context of large organisations to small and medium sized companies in order to investigate the consequences of the use of Web 2.0 technologies on multiple cases of e-learning companies. The study finds that the ability of companies to cope with the potential disruption caused by such technologies depends on the quality of their leadership which is related to the age of the organization and its risk profile.

In his chapter on "Two paths for innovation: Parvenu or Pariah", Bryant aims to cast doubt on much of the conventional wisdom concerning entrepreneurial creativity and innovation. Drawing on a rich background of economics and philosophy of science, he shows that the debate surrounding entrepreneurship is dangerously one-sided. Creativity and innovation are not and should not be limited to market interaction. Other aspects of society show aspects of creativity and innovation, and might indeed lead the way for commercial organisations to follow. Decades of unbridled market worship have, however, rendered these views relatively irrelevant, to the disadvantage of society in general, but also of businesses themselves. Bryant uses some of the current economical woes to support his argument that a broader understanding of innovation and creativity will be conducive in overcoming some of the societal issues we face.

Following from Bryant's critical evaluation of current discourses, Venable offers a similarly critical view of methodology in his "Identifying and Addressing Stakeholder Interests in Design Science Research: An Analysis Using Critical Systems Heuristics". He builds on the current debate surrounding design science research and explores the role that Critical Systems Heuristics can play in moving design science research forward. This chapter stands in the tradition of critical research that has often been the centre of attention in IFIP WG 8.2 work but it combines this critical tradition with an eminently practical intention. Using the ideas of Critical Systems Heuristics, Venable points out that design cannot and should not be exclusively geared towards the interests of those in power but that the whole range of stakeholders affected by a technology under development should be considered. The critical angle allows him to develop a philosophically and theoretically grounded framework that can inform stakeholder development in systems design. It addresses the difficult question of the representation of stakeholders who are unable to participate directly and introduces the idea of using witnesses in design processes.

Mamede and Santos address the question of creativity and innovation in IS from a very different angle. By commenting on "An Architecture for a Creative Innovation System", they move the focus away from human and organizational issues and open the discussion to include the question whether information systems themselves can be creative. The concept of creativity, they explore, is that of random word generation, also called brute thinking, which aims to develop new angles of problems that allow users to come up with new answers. The authors then go on to develop the architecture of a process that would allow the implementation of a system, fulfilling their definition of a creative information system.

The theme of creativity in systems design is then developed in a different direction in the last chapter of the section. Conboy, Wang and Fitzgerald look at current research 
on creativity in agile systems development. Their starting point is the proposition that agile methods foster creativity and there is lack of empirical research to support this assumption. The development of agile methods has been driven by the practitioner community, leaving questions of rigorous validation open, particularly in the area of SMEs. Using the theoretical lens of creativity theory, the authors discuss the extent to which creativity in agile projects actually occurs. The results of the study show gaps in current knowledge and points towards promising areas of further research.

\section{Challenges in Ensuring Effective Governance}

Governance relates to organizational processes dealing with internal control and ensuring effective utilization of information. Well-designed information systems are central to good governance. In many ways, correctness in system specification and the related organizational design helps ensure the establishment of good responsibility and accountability structures, which are central to good governance. In terms of SMEs, there are a number of governance challenges that need to be carefully understood and articulated. Some of these are addressed by the papers in this conference.

Devos, Landeghem and Deschoolmeester in their paper "IT Governance in SMEs: Trust or control?' start with the observation that it is futile to apply theories of IT governance which are relevant to large firms to SME's as the realities of both are diametrically opposite. In their paper, the authors try to address the very pertinent question of the reasons and manifestation of outsourced information system failure (OISF) in an SME. They try to bring this to light by qualitative and positivistic IS case study research, which delves into the on-ground scenario of as many as eight different cases of IS projects. They offer the premise that in SME's, theory of trust, fairness, intuition and empathy are more relevant and provide better guidelines for IT governance. This study sheds light on an aspect which has been widely accepted, but rarely documented - that trust and personal equations are the bedrock of systematic and procedural guidelines in SME's where people are more closely aligned with each other's aspirations, personality traits and operate with much more intimacy than is possible in a large organisation. The authors validate this understanding and also advise future researchers to hone on SME's specifically in their work, instead of drawing conclusions from observations made in large firms, which are largely redundant when applied to SME's.

Mamede and Amaral in their "Methodology for Electronic Business Initiatives" point out that the traditional systems development methodologies displays weaknesses that render them problematic, in particular for small and medium-sized organizations. In order for SMEs to make use of the potential offered by web-based technology, methodologies more specifically aimed at their needs are required. The authors use the chapter to argue for a methodology that is centred on the implementation lifecycle of electronic business in SMEs.

Taking this argument forward are Huang, Zmud and Price in their paper "IT Governance Practices in Small and Medium-Sized Enterprises: Recommendations from an Empirical Study", where they also contend that there is much documentation available about the concentration, flow and direction of decision-making in large organizations. While the need for better understanding of the same contexts in SME's is palpable, 
documentation and observation about these is lamentably lacking in the case of SME's. This leaves the field wide open for researchers, who must track, observe and record the shape and flow of decisions in small firms where the decision making authority is often confined to a group of individuals comprising the top management. The interesting facets of decision making, which this phenomenon results in, have been recorded through interviews with top managers from three SME's. Based on the insights gained from these interactions, the authors make thought provoking and pertinent recommendations, which invite further scrutiny and follow-ups.

It is indisputable that the high winds of current economic realities have greatly buffeted the mechanisms, which ensure effective governance across organisations. These have been more sharply felt in small firms where cost cutting has directly impacted IT processes by eroding into the investments on IT and decrease in the manpower running IT processes. There is little debate that this factor has caused a lot of disruption in the overall efficiency, market worthiness and competitiveness of the enterprise. In such a scenario, Albayrak and Gadatschhave tackled this pressing issue by presenting a reference model for IT controlling, "Life cycle Model for IT Performance Management: A reference model for small and medium enterprises". This model has the potential to bail out many a small firm embroiled in arbitrary cost cutting. It is founded on a performance oriented framework which is based on that most required but increasingly rare commodity-common sense. In the present economic situation, with resources drying up, this inexhaustible and germane commodity can prevent many firms from sacrificing efficiency and performance on the altar of forced economy. The authors present their model to be used in the organizations where it is most required and likely to succeed- small and medium firms.

\section{Challenges in Managing Knowledge}

The definition of knowledge as true justified beliefs is often ascribed to Plato. From the perspective of the scholar who is interested in knowledge management in SMEs this definition can offer the advantage of opening up new avenues of inquiry. Rather than getting caught in the difficult question of whether knowledge can be subject to management in the first place, one can look at more established epistemological issues of truth and justification and how these are affected by a particular organizational environment, such as an SME. Knowledge management is often taken for granted as a positive option that organizations should avail themselves of. This is somewhat surprising due to the conceptual uncertainty that surrounds it and much empirical research that sheds doubt on its effectiveness. If we are to understand knowledge management better, then research is needed into the conceptual basics as well as the organizational realities where the conceptual issues are filled with life. The chapters in this section all contribute to this and use an empirical study to investigate how knowledge can be shared and managed in SMEs.

In their "Knowledge Management in Small Firms", Panyasorn, Panteli and Powell describe experiences of the use of a particular technology, Lotus Notes, as a means to facilitate knowledge management in a developing country. This study aims to provide a counterpoint to the dominant focus on larger enterprises in IS and knowledge management research. They use an interpretive approach to develop three case studies. On 
the basis of the data they derive from this approach, they come up with taxonomy of the use of technology in different organizational processes that leads to or constitutes management of knowledge. The authors find evidence of knowledge creation on a lower hierarchical level whereas no such evidence is found at the organizational level. According to their structure, smaller companies can make better use of some technologies such as groupware than larger ones.

The subsequent chapter by Ramos, Cardoso and Carvalho offers further empirical evidence of the relevance of knowledge management to SMEs by exploring the idea of open innovation. A recurring theme of this book, namely that SMEs find it more difficult to institute regular $\mathrm{R} \& \mathrm{D}$ processes due to the smaller amount of resources they can dedicate to this, is addressed using the idea of open innovation. The chapter describes a piece of action research undertaken at the University of Minho with the aim of exploring how SMEs could be enabled to benefit from innovation developed elsewhere, i.e. in universities. Transfer of knowledge and technology, while politically desired by most government runs into a range of problems, from communication and technology to intellectual property regimes. Ramos et al. describe the development of a business model and a technology platform for an innovation broker that aims to connect ideas and technologies from a university to potential SME users. The chapter offers an evaluation of this strategy which may serve as a role model for future knowledge transfer.

The final chapter in the section on knowledge management and SMEs focuses on recent technological developments, notably on web 2.0 technologies. In his "Services Supporting Knowledge Maturing in Small and Medium-Sized Enterprises", Maier discusses reasons why much-hyped communication technologies have not yet been used successfully in knowledge management in SMEs. He argues that a general theoretical interest has not translated into practical guidance on how such technologies can be employed to address the knowledge needs of SMEs. Building on the theoretical basis of a knowledge-maturing model, the chapter offers the findings of an ethnographic study into the topic. The results are conducive to practical use in that they offer organizations ways in which to engage in knowledge sharing and cooperation with the main stakeholders of the organization.

\section{Organizational Challenges, Power and Politics}

SMEs are faced with a number of organizational challenges. While most SMEs may be in a rather steady state, aspiring to address the needs of their customers in an effective manner, there are an increasing number of SMEs that are innovative and responsive enough to be termed 'high growth' firms. Current research into SMEs suggests that there are a number of factors that come together to ensure their relative success or failure. Owner-manager characteristics and motivation is clearly an aspect that determines the nature and scope of leadership that a given SME might have. The nature of the business also plays an important role in defining SME challenges. For instance, in the traditional manufacturing enterprise, the power of large firms over smaller ones may be a significant influencer on their performance. In other cases however SMEs may be instrumental in defining the characteristic of a new market. Business characteristics, strategic influences and external environment may also define the direction of a given SME. 
There is some similarity between the challenges that a SME, large enterprise or a government institution may face. Inadequacy of systems requirement assessment and an inability to integrate various user perspectives certainly causes problems. Wenger et al (2005) discuss how the implementation of an ERP system at a SME got off to a bad start when the owner-managers began using the system as a means of organizational restructuring. This led to different kinds of power getting manifested in the enterprise. Similarly, Yan and Panteli in their paper, "The Emergence of 'Power With': The Case of a Born Global Organization" discuss various organizational power issues with respect to Born Global organizations. Born Global enterprises are defined as businesses that are global, yet local, small but with a global outreach in terms of new product and services. Such enterprises have also been referred to as lean, mean and agile.

Along similar lines Wastell, White and Broadhurst in their paper "The Chiasmus of Design: paradoxical outcomes in the e-Government reform of UK Children's Services" presents an ethnography using McLuhan's concept of chiasmus. The study presents paradoxical outcomes of the use of an Integrated Children's System, where the intended outcome gets shelved and spawns contradictory effects. The flawed design and requirements assessments, as was also observed by Wenger et al (2005), resulted in compromises in record keeping, investigative approaches and generally a high level of risk. The paper ends by proposing an alternative approach to design, based on socio-technical precepts, emphasizing the principles of minimum critical specification, user-centeredness and local autonomy.

Other related challenges include aspects of technology acceptance, particularly evaluating factors that facilitate acceptance of systems in SMEs. At times it is perhaps a difference in perceptions that leads to or inhibits uptake of a given system. While SMEs, to some extent, present a collective mind, yet there are factors that enable successful adoption or non-adoption of systems. Many entrepreneurs recognize the challenges in SMEs and information systems and hence venture into establishing such an enterprise.

This intriguing aspect also forms the basis for Mourmant's study of "How and why do IT entrepreneurs leave their salaried employment to start a SME?- A mixed methods research design". The author sets himself a tall task and one, which has gained a lot of relevance and visibility in recent times. He explores the reasons and motivations that compel salaried IT professionals to leave the comparative security of their well paid jobs and venture into the untried and turbulent waters of entrepreneurship. The author has developed a conceptual framework that begins with the explanation and rebuttal of two streams of extant research. The first of these two streams refer to the literature pertaining to entrepreneurship, which is already developed and the second alludes to the characteristics and attributes of IT industry as a whole which significantly impacts employee turnover. From the vantage point of an observer seeking to note and record the exigencies that the present economic scenario necessitates, Mourmant forwards the argument that employee turnover is directly related and proportional to prevailing market conditions. He concludes his study with suggestions for IT managers and entrepreneurs to be able to cope better in trying times.

Continuing with the theme of power and politics present in organization, Costello and Moreton present their research in "Towards a Model of Technology Adoption: A Conceptual Model Proposition”. They contend that Information Communication 
Technology (ICT) practitioners in small and medium firms face the same issues and challenges which their larger counterparts face. The authors prepare a case for governmental intervention, which can facilitate the adoption of better and more efficient ICT. The present weaknesses in the ICT adoption are easily discernable and a new framework is presented which promises to provide an enhanced understanding of the ways in which ICT usage can be increased and improved.

\section{Epilogue}

Innovation and creativity are fundamental to the survival of SMEs. We are pleased that the range of papers being presented at the IFIP 8.2 creativeSME conference offer interesting insights into these two areas. There is no doubt that innovativeness nurtures creativity, which in turns allows SMEs to define distinctive competencies that are so necessary in a competitive marketplace. As has been recognized by many authors in these proceedings, majority of the research in innovativeness and creativity has remained focused on large corporations and relatively less in the context of SMEs. We are hopeful that the IFIP 8.2 creativeSME conference will set the tone for an interesting discourse in this rather important area. Some of the papers identified significant future research directions. For instance aspects of process integrity, organizational power, politics and information security have been identified. We are certain that many such issues will be discussed at this conference and beyond.

\section{References}

Boden, M.A.: Computing and Creativity. In: Baird, R.M., Ramsower, R., Rosenbaum, S.E. (eds.) Cyberethics - Social and Moral Issues in the Computer Age, pp. 308-319. Prometheus Books, New York (2000)

Cooper, R.B.: Information technology development creativity: A case study of attempted radical change. MIS Quarterly 24(2), 245-275 (2000)

Datta, P.: An Agent-Mediated Knowledge-in-Motion Model. Journal of the Association for Information Systems 8(5), 211-287 (2007)

Lessig, L.: The Future of Ideas - the Fate of the Commons in a Connected World. Vintage, New York (2001)

Rogers, E.M.: Diffusion of Innovations, 5th edn. The Free Press, New York (2003)

Wenger, M., Dhillon, G., Caldeira, M.: ERP Implementation in Portugal: interpreting dimensions of power. In: AMCIS, Omaha (2005) 